\section{FRI0188 EARLY RENAL IMPROVEMENTS FOLLOWING RITUXIMAB IN LUPUS NEPHRITIS}

Sophie Collinson, Ben Parker, Eoghan Mccarthy, lan N. Bruce, British Isles Lupus Assessment Group Biologics Register. Kellgren Centre for Rheumatology, NIHR Manchester Musculoskeletal Biomedical Research Centre, Manchester University Hospitals NHS Foundation Trust, Manchester Academic Health Science Centre, Manchester, United Kingdom

Background: Rituximab (RTX) is the monoclonal antibody of choice in patients with lupus nephritis (LN) who either fail to respond to, or cannot receive standard of care (SOC) therapy. Despite its continued use in clinical practice, published clinical trials regarding its use in LN management have failed to show any clear benefit.

Objectives: We aimed to analyse renal outcomes of $L N$ patients in a large national register.

Methods: Patients with active LN on recruitment into the BILAG-Biologics Register (BILAG-BR) were included. Active LN was defined as a BILAG A or B score in the renal domain. Patients are eligible for RTX if they have failed either MMF or cyclophosphamide. We defined renal response at 6 and 12 months as a change in BILAG score from $A$ to $B / C / D$ or $B$ to $\mathrm{C} / \mathrm{D}$. Non-renal disease activity was also described.

Results: Overall 259/897 patients in BILAG-BR had active LN at baseline. Of these, 230 received RTX and 29 received SOC. 109/159 (68.6\%) RTX patients and $15 / 19(78.9 \%)$ of SOC patients had a renal BILAG response at 6 months $(p=0.792)$ (Table 1). In those with sufficient response data at 12 months, $31 / 98(31.6 \%)$ and $7 / 15$ (46.7\%) ( $p=0.084)$ demonstrated continued renal response in the RTX and SOC groups respectively. Both groups made improvements or remained stable in all other renal domains (UPCR, eGFR, creatinine) at 6 and 12 months. Oral steroid doses decreased in both treatment groups. Of note, baseline steroid doses in the SOC group were higher than RTX patients $30 \mathrm{mg}(20-$ $40)$ vs. $12.5 \mathrm{mg}(10-25), \quad \mathrm{p}<0.001)$. Non-renal disease activity measures improved in both cohorts.

\begin{tabular}{|c|c|c|c|}
\hline Variable & RTX & SOC & $\mathbf{p}$ \\
\hline Renal BILAG response* & $109 / 159$ & $15 / 19(78.9 \%)$ & 0.792 \\
\hline - $6 \mathrm{~m}(n=159$ RTX, 19 SoC) & $(68.6 \%)$ & $7(46.7 \%)$ & 0.084 \\
\hline - 6 to $12 \mathrm{~m}(n=98 R T X, 15 \mathrm{SoC})$ & $31(31.6 \%)$ & & \\
\hline UPCR (median [IQR]) $\mathrm{mg} / \mathrm{mmol}$ & $215(103-388)$ & $173(88-352)$ & 0.2945 \\
\hline$-0 \mathrm{~m}(n=160)$ & $149(54-404)$ & $66(21-92)$ & 0.331 \\
\hline$-6 m(n=92)$ & $71(42-316)$ & $18(11-40)$ & 0.0045 \\
\hline \multicolumn{4}{|l|}{$-12 m(n=60)$} \\
\hline Serum creatinine (median [IQR]) micromol/ & $69(58-97)$ & $66(52-90)$ & 0.3864 \\
\hline 1 & $70(59-99)$ & $70(51-84)$ & 0.4774 \\
\hline$-0 m(n=239)$ & $70(59-100)$ & $68(60-95)$ & 0.7651 \\
\hline \multicolumn{4}{|l|}{$-6 m(n=157)$} \\
\hline \multicolumn{4}{|l|}{$-12 m(n=105)$} \\
\hline Oral steroid dose (median [IQR]) mg/day & $12.5(10-25)$ & $30(20-40)$ & $<0.001$ \\
\hline - Om (n=196 RTX, 29 SoC) & $10(7-17.5)$ & $12.5(10-20)$ & 0.1246 \\
\hline$-6 \mathrm{~m}(n=151 R T X, 19$ SoC) & $10(5-15)$ & $8.5(6.25-$ & 0.9730 \\
\hline$-12 \mathrm{~m}(n=108 \mathrm{RTX}, 16 \mathrm{SoC})$ & & 12.5) & \\
\hline Non-renal BILAG & $9(1-12)$ & $8(1-10)$ & 0.9310 \\
\hline$-0 m(n=230)$ & $1(0-2)$ & $1(0-2)$ & 0.6527 \\
\hline$-6 m(n=153)$ & $1(0-2)$ & $1(0-2)$ & 0.7803 \\
\hline \multicolumn{4}{|l|}{$-12 m(n=101)$} \\
\hline Non-renal SLEDAI (median [IQR]) & $3(2-4)$ & $2(1-3)$ & 0.0426 \\
\hline - $0 \mathrm{~m}(n=254)$ & $1(0-2)$ & $1(0-2)$ & 0.2845 \\
\hline$-6 m(n=183)$ & $1(0-2)$ & $1(0-2)$ & 0.6887 \\
\hline \multicolumn{4}{|l|}{$-12 m(n=113)$} \\
\hline SLICC DI $\geq 1$ & $113(50.2 \%)$ & $14(48.3 \%)$ & 0.844 \\
\hline$-0 m(n=254)$ & & & \\
\hline
\end{tabular}

Conclusion: In a large UK-wide, real-world, observational LN cohort we have shown that RTX does improve renal outcomes at 6 and 12 months in refractory SLE patients. RTX is a valid treatment option for many patients with renal disease in SLE.

Disclosure of Interests: Sophie Collinson: None declared, Ben Parker Grant/research support from: GSK, Consultant for: AZ, UCB, GSK, Eoghan McCarthy: None declared, lan N. Bruce Grant/research support from: Genzyme Sanofi, GlaxoSmithKline, Consultant for: AstraZeneca, Eli Lilly, GlaxoSmithKline, ILTOO Pharma, Medlmmune, Merck Serono, Speakers bureau: GlaxoSmithKline, UCB Pharma

DOI: 10.1136/annrheumdis-2019-eular.317

\section{FRI0189 POOLED ANALYSIS OF THE REAL-WORLD} EFFECTIVENESS OF BELIMUMAB IN TREATMENT OF SYSTEMIC LUPUS ERYTHEMATOSUS USING MULTICOUNTRY DATA FROM THE OBSERVE STUDIES

Christopher Collins ${ }^{1}$, Josefina Cortés-Hernández ${ }^{2}$, Mercedes García ${ }^{3}$, Johannes von Kempis ${ }^{4}$, Andreas Schwarting ${ }^{5}$, Zahi Touma ${ }^{6}$, Shannon Ferrante ${ }^{7}$, Milena Kurtinecz ${ }^{7}$, Kerry Gairy. ${ }^{8}$ Washington Hospital Center, Washington, United States of America; ${ }^{2}$ Vall d Hebron Hospital, Barcelona, Spain; ${ }^{3}$ HIGA San Martin La Plata, La Plata, Argentina; ${ }^{4}$ Kantonsspital St. Gallen, St Gallen, Switzerland; ${ }^{5}$ Johannes Gutenberg-University, Mainz, Germany; ${ }^{6}$ Toronto Western Hospital, University of Toronto Lupus Clinic, Toronto, Canada; ${ }^{7}$ GSK, Collegeville, United States of America; ${ }^{8}$ GSK, Brentford, United Kingdom

Background: Real-world evidence of belimumab effectiveness in patients with systemic lupus erythematosus (SLE) has been reported separately for several countries through the OBSErve (evaluation Of use of Belimumab in clinical practice SEttings) programme.

Objectives: To evaluate the effectiveness of belimumab in patients with SLE using pooled data from the individual OBSErve studies.

Methods: This was a post hoc meta-analysis (GSK study 206351) of patient level data pooled from six retrospective observational cohort studies (Argentina, Canada, Germany, Spain, Switzerland, United States of America). Physicians provided data for adults ( $\geq 18$ years; clinical diagnosis of SLE) who had initiated intravenous belimumab as part of their usual SLE care $\geq 6$ months prior to enrolment and for whom reasons for belimumab initiation could be identified. Primary objective: physicianassessed overall clinical response to belimumab at Month 6, which reflected physicians' impression of change in overall clinical manifestations, categorised as worse, no improvement, and improvement of $<20 \%$, $20-49 \%, 50-79 \%$, and $\geq 80 \%$. Secondary objectives included a description of patient characteristics at belimumab initiation (index); treatment patterns during the 6 months after index; and patient and treatment characteristics associated with an overall clinical response. Safety was not assessed. Results: Data were pooled from all 830 patients $(89.3 \%$ female; mean [standard deviation] age 41.9 [12.6] years) included in each of the OBSErve studies. At index, most patients had moderate SLE $(71.4 \%$; physician-assessed) and serological indicators of high disease activity (82.8\%; high anti-dsDNA and/or low complement). Of 345 patients for whom Safety of Estrogens in Lupus Erythematosus-National Assessment Trial-SLE Disease Activity Index baseline data were available, $60.0 \%$ had a score $>10$. At index, $72.0 \%$ of patients had musculoskeletal, $59.0 \%$ had mucocutaneous manifestations, and $16.5 \%$ had renal involvement. At Month $6,82.8 \%$ of patients had $>20 \%$ improvement and $48.1 \%$ had $\geq 50 \%$ improvement in their overall condition; only $4.7 \%$ had none or a worse response to belimumab therapy (Figure). At index, $81.4 \%$ of patients received steroids at a mean dose of $17.6 \mathrm{mg} /$ day. At Month 6 , the mean change in steroid dose was $-9.7 \mathrm{mg} /$ day. Of those receiving a dose $>7.5 \mathrm{mg} /$ day $(78.4 \%$ of all patients receiving steroids at index), $54.5 \%$ had a dose reduction to $\leq 7.5 \mathrm{mg} /$ day at Month 6 . Ineffective previous treatment was the main reason for belimumab initiation $(74.3 \%)$. Prior to Month $6,4.4 \%$ of patients $(n=12 / 270)$ in Argentina, Spain, Germany and Switzerland discontinued belimumab, most frequently due to lack of efficacy and patient request (both $33.3 \%$ ); OBSErve US and Canada excluded patients with $<6$ months' belimumab treatment.

Conclusion: This study provides important insights into global real-world outcomes in a large number of patients with SLE treated with belimumab. After 6 months of treatment, most patients demonstrated clinical improvements in SLE; results were consistent across the pooled population and for patients with high disease activity. Belimumab was steroid sparing; most patients receiving steroids at belimumab initiation decreased their steroid dose after 6 months of belimumab treatment.

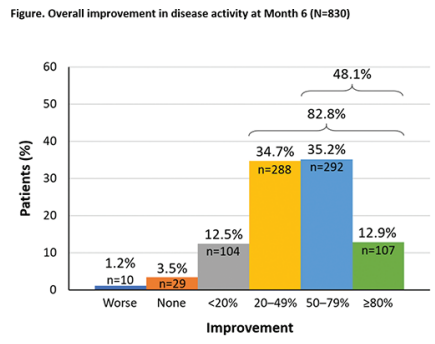

